\title{
Polarization effects in the search for a dark vector boson at $e^{+} e^{-}$colliders
}

\author{
Fei-Fan Lee $\odot,{ }^{1}$ Guey-Lin Lin $\odot,{ }^{2}$ and Vo Quang Nhat $\odot^{2}$ \\ ${ }^{1}$ Department of Physics, Jimei University, 361021 Xiamen, Fujian province, People's Republic of China \\ ${ }^{2}$ Institute of Physics, National Chiao Tung University, Hsinchu 30010, Taiwan
}

(Received 25 August 2020; accepted 26 December 2020; published 12 January 2021)

\begin{abstract}
We argue that the search for dark vector boson through $e^{+} e^{-} \rightarrow Z_{d} \gamma$ can determine the Lorentz structure of $Z_{d} l^{+} l^{-}$couplings with the detection of leptonic decays $Z_{d} \rightarrow l^{+} l^{-}$. We assume a general framework that the dark vector boson interacts with ordinary fermions through vector and axial-vector couplings. As a consequence of Ward-Takahashi identity, $Z_{d}$ is transversely polarized in the limit $m_{Z_{d}} \ll \sqrt{s}$. On the other hand, the fraction of longitudinal $Z_{d}$ is non-negligible for $m_{Z_{d}}$ comparable to $\sqrt{s}$. Such polarization effects can be analyzed through angular distributions of final-state particles in $Z_{d}$ decays. Taking $l^{ \pm} \equiv \mu^{ \pm}$, we study the correlation between $Z_{d}$ angle relative to $e^{-}$beam direction in $e^{+} e^{-}$center of momentum frame and $\mu^{-}$angle relative to the boost direction of $Z_{d}$ in $Z_{d}$ rest frame. This correlation is shown to be useful for probing the Lorentz structure of $Z_{d} l^{+} l^{-}$couplings. We discuss the measurement of such correlation in Belle II detector, taking into account the detector acceptance and energy resolution.
\end{abstract}

DOI: 10.1103/PhysRevD.103.015016

\section{INTRODUCTION}

Searching for dark matter (DM) is one of the major endeavors in the present day particle physics community. The efforts in direct and indirect detections as well as productions of DMs in LHC so far have not produced positive results. Recently, there are growing interests to search for DM related phenomena with huge statistics and high precision measurements. These phenomena involve the hidden sector [1-6], which is assumed to interact with Standard Model (SM) particles through certain messengers. A popular proposal for such a messenger particle is the socalled dark photon, which mixes with the $U(1)$ hypercharge field $B_{\mu}$ in SM,

$\mathcal{L}_{\text {gauge }}=-\frac{1}{4} B_{\mu \nu} B^{\mu \nu}+\frac{1}{2} \frac{\varepsilon_{\gamma}}{\cos \theta_{W}} B_{\mu \nu} A_{\mu \nu}^{\prime}-\frac{1}{4} A_{\mu \nu}^{\prime} A^{\prime \mu \nu}$,

where $A_{\mu}^{\prime}$ is the dark photon field and $A_{\mu \nu}^{\prime} \equiv \partial_{\mu} A_{\nu}^{\prime}-\partial_{\nu} A_{\mu}^{\prime}$. The above mixing induces electromagnetic couplings, $\mathcal{L}_{\mathrm{em}}=\varepsilon_{\gamma} e J_{\mathrm{em}}^{\mu} A_{\mu}^{\prime}$, between the dark photon and SM fermions, which generate rich phenomenology [7]. On the other hand, the neutral current couplings between the same set of particles are further suppressed by the factor $m_{A^{\prime}}^{2} / m_{Z}^{2}$ for $m_{A^{\prime}} \ll m_{Z}$ with $m_{A^{\prime}}$ the dark photon mass. However,

Published by the American Physical Society under the terms of the Creative Commons Attribution 4.0 International license. Further distribution of this work must maintain attribution to the author(s) and the published article's title, journal citation, and DOI. Funded by SCOAP ${ }^{3}$. independent neutral current couplings can be generated through mass mixing between the messenger particle and the $Z$ boson [8-10]. In this case, the messenger particle is often referred to as $Z^{\prime}$ boson. The mass mixing term $\delta m^{2} Z_{\mu}^{\prime} Z^{\mu}$ can induce neutral current couplings $\mathcal{L}_{\mathrm{NC}}=$ $\left(g \varepsilon_{Z} / \cos \theta_{\mathrm{W}}\right) J_{\mathrm{NC}}^{\mu} Z_{\mu}^{\prime}$ with $\varepsilon_{Z} \equiv \delta m^{2} / m_{Z}^{2}$. For a general scenario that both kinetic and mass mixings are present, the interactions between dark boson and SM fermions are given by

$$
\mathcal{L}_{\text {int }}=\left(\varepsilon_{\gamma} e J_{\mathrm{em}}^{\mu}+\varepsilon_{Z} \frac{g}{\cos \theta_{\mathrm{W}}} J_{\mathrm{NC}}^{\mu}\right) Z_{d, \mu},
$$

with $Z_{d}$ the dark boson, which is the generalization of $A^{\prime}$ and $Z^{\prime}{ }^{1}$

We note that the physical masses of $Z_{d}$ and $Z$ are given by diagonalizing of the following mass matrix:

$$
\begin{aligned}
\mathbf{M}_{\mathbf{0}}^{\mathbf{2}} & =\left(\begin{array}{cc}
m_{Z^{0}}^{2} & -\varepsilon_{Z} m_{Z^{0}}^{2} \\
-\varepsilon_{Z} m_{Z^{0}}^{2} & m_{Z_{d}^{0}}^{2}
\end{array}\right) \\
& +\left(\begin{array}{cc}
0 & -\varepsilon_{\gamma} \tan \theta_{\mathrm{W}} m_{Z^{0}}^{2} \\
-\varepsilon_{\gamma} \tan \theta_{\mathrm{W}} m_{Z^{0}}^{2} & \left(2 \varepsilon_{\gamma} \varepsilon_{Z} \tan \theta_{\mathrm{W}} m_{Z^{0}}^{2}+\varepsilon_{\gamma}^{2} \tan \theta_{\mathrm{W}}^{2} m_{Z^{0}}^{2}\right)
\end{array}\right),
\end{aligned}
$$

where the second term arises from the diagonalization of kinetic Lagrangian $\mathcal{L}_{\text {gauge }}$. To ensure the positivity of

\footnotetext{
${ }^{1}$ Strictly speaking, $\varepsilon_{Z}$ in Eq. (2) is $\delta m^{2} / m_{Z^{0}}^{2}$ with $m_{Z^{0}}$ the $Z$ boson mass before mass matrix diagonalization. Nevertheless, for numerical calculations, we take $\varepsilon_{Z} \equiv \delta m^{2} / m_{Z}^{2}$ as the correction is of higher orders.
} 
physical masses, one requires the determinant of $\mathbf{M}_{0}^{2}$ be positive, i.e., $\operatorname{det}\left(\mathbf{M}_{\mathbf{0}}^{\mathbf{2}}\right)=\left(m_{Z_{d}^{0}}^{2} m_{Z^{0}}^{2}-\left(\delta m^{2}\right)^{2}\right)>0$. We note that the second term of the mass matrix does not give additional contribution to the determinant. This condition constrains models that generate masses for $Z, Z_{d}$, and the mass mixing between the two. For our interested scenario $\varepsilon_{Z} \simeq \varepsilon_{\gamma}$ with the latter in the range $\left(10^{-3}-10^{-4}\right)$, i.e., current limits from dark photon searches at $e^{+} e^{-}$colliders, $m_{Z_{d}^{0}}$ should be greater than (10-100) MeV. In Ref. [9], two Higgs doublets and one scalar Higgs singlet are introduced such that $m_{Z^{0}}^{2}=g^{2}\left(v_{1}^{2}+v_{2}^{2}\right) /\left(4 \cos ^{2} \theta_{\mathrm{W}}\right), m_{Z_{d}^{0}}^{2}=$ $g_{d}^{2}\left(v_{2}^{2}+v_{d}^{2}\right)$, and $\delta m^{2}=g_{d} g v_{2}^{2} /\left(2 \cos \theta_{\mathrm{W}}\right)$. Here $g_{d}$ is the dark boson gauge coupling to the second Higgs doublet and the scalar Higgs singlet, while $v_{1}\left(v_{2}\right)$ and $v_{d}$ are vacuum expectation values of neutral scalar in the first (second) Higgs doublet and that of the scalar Higgs singlet. The above-mentioned lower limit on $m_{Z_{d}}$ requires $g_{d}>4 \times$ $\left(10^{-5}-10^{-4}\right)$ if $\left(v_{d}^{2}+v_{2}^{2}\right) \simeq\left(v_{1}^{2}+v_{2}^{2}\right) \simeq(246 \mathrm{GeV})^{2}$.

The search for the light vector boson with the reaction $e^{+} e^{-} \rightarrow Z_{d} \gamma$ has been proposed before [11-13]. Particularly, there exist phenomenological studies on dark sectors under the environment of $e^{+} e^{-}$colliders [14-22]. Along this line, the experimental searches for $Z_{d}$ proceed through the detection of visible and invisible $Z_{d}$ decays. The visible mode requires a full reconstruction of $Z_{d}$ peak through measuring the energy and momentum of lepton or light hadron pairs from $Z_{d}$ decays [23-28], while the invisible mode looks for the peak of missing mass at $m_{Z_{d}}$ given by $M_{\text {mass }}^{2}=\left(P_{e^{-}}+P_{e^{+}}-P_{\gamma}\right)^{2}$ [29]. We note that both phenomenological and experimental studies mentioned above consider only the dark photon scenario, i.e., $Z_{d}$ interacts with $\mathrm{SM}$ fermions only via electromagnetic current. On the other hand, since neutral-current coupling is also possible, it is of great importance to simultaneously detect $Z_{d}$ and measure the Lorentz structure of its coupling to SM fermions. To determine the relative strengths of vector and axial-vector couplings, such as the ratio $g_{f, A} / g_{f, V}$ in the generic structure $e \varepsilon \bar{f}\left(g_{f, V} \gamma_{\mu}+g_{f, A} \gamma_{\mu} \gamma_{5}\right) f Z_{d}^{\mu}$, it is necessary to measure the angular distributions of final-state fermions from $Z_{d}$ decays. ${ }^{2}$

The dark vector boson $Z_{d}$ produced by $e^{+} e^{-} \rightarrow Z_{d} \gamma$ is polarized. In fact, $Z_{d}$ must be in one of the transversely polarized states in the limit $\sqrt{s} \gg m_{Z_{d}}$. This is a direct consequence of Ward-Takahashi identity [30] to be elaborated in the next session. Furthermore, with the presence of both $g_{f, A}$ and $g_{f, V}$, parity symmetry is broken. Hence, there exists a forward-backward asymmetry for the production of each transversely polarized $Z_{d}$ state, while the production of longitudinal $Z_{d}$ is forward-backward symmetric. The magnitude of the above asymmetry is directly

\footnotetext{
${ }^{2}$ Here we choose the normalization $g_{f, V}^{2}+g_{f, A}^{2}=1$.
}

related to the degree of parity violation, characterized by the parameter $\rho \equiv 4 g_{f, A} g_{f, V}$ under the normalization $g_{f, V}^{2}+g_{f, A}^{2}=1$. For a fixed $\rho$, the asymmetry reaches to the maximum for $m_{Z_{d}} / \sqrt{s} \rightarrow 0$. Besides the asymmetry in the production of transversely polarized $Z_{d}$ state, there is also forward-backward asymmetry for the angular distributions of final-state fermions from $Z_{d}$ decays, which is also controlled by the same parameter $\rho$. Hence, the correlation between these two asymmetries can be exploited to probe $\rho$.

The most sensitive search for $Z_{d}$ through the visible mode $e^{+} e^{-} \rightarrow Z_{d} \gamma \rightarrow e^{+} e^{-} \gamma, \mu^{+} \mu^{-} \gamma$ is performed by $B A B A R$ [24]. Using $514 \mathrm{fb}^{-1}$ of data, the upper limits on the mixing parameter $\varepsilon$ is $10^{-4}-10^{-3}$ for $m_{Z_{d}}$ between 0.02 and $10.2 \mathrm{GeV}$. Comparable sensitivity to $\varepsilon$ is expected at Belle II [31-34] with $500 \mathrm{fb}^{-1}$ of integrated luminosity. Belle II is an electron-positron collider experiment running at the SuperKEKB accelerator. It is a next-generation Bfactory experiment aiming to record a data set of $50 \mathrm{ab}^{-1}$. In this paper, we focus on the prospect of detecting $Z_{d}$ and measuring the parity violation parameter in its interaction with SM fermions with $e^{+} e^{-} \rightarrow Z_{d} \gamma$ followed by $Z_{d} \rightarrow$ $\mu^{+} \mu^{-}$decay at Belle II. Backgrounds to this process are QED process $e^{+} e^{-} \rightarrow \mu^{+} \mu^{-} \gamma^{3}$ and the resonant production process $e^{+} e^{-} \rightarrow \gamma X[X=J / \psi, \psi(2 S), \Upsilon(1 S), \Upsilon(2 S)]$ followed by $X \rightarrow \mu^{+} \mu^{-}$. We will not consider the decay mode $Z_{d} \rightarrow e^{+} e^{-}$in this paper since backgrounds to this mode are more complicated, including $e^{+} e^{-} \rightarrow e^{+} e^{-}(\gamma)$ and $e^{+} e^{-} \rightarrow \gamma \gamma(\gamma)$. Since we are mainly interested in probing the parity violation effect by $Z_{d} f \bar{f}$ coupling, the study on $Z_{d} \rightarrow \mu^{+} \mu^{-}$is sufficient to make our point. We note that there are recent interests in the signals for $17 \mathrm{MeV}$ protophobic vector boson [35-37] motivated from anomalies in ${ }^{8} \mathrm{Be}$ and ${ }^{4} \mathrm{He}$ nuclear transitions [38,39]. Searching for vector boson in $e^{+} e^{-}$colliders for this particular parameter range has been proposed [40,41]. Although we shall not focus on such a specific scenario, we do notice that the protophobic vector boson interacts with the electron through both vector and axial-vector couplings. However, parity violation effects resulting from the presence of both couplings were not considered in the above analyses. For an earlier candidate of light neutral gauge boson [42], its parity-violating effects to low energy neutral current processes were studied quite some time ago [43]. Among those low energy processes, the search for atomic parity violations [44,45] still attracts high attentions in recent years. For phenomenological discussions on this issue under the current dark boson scenario, see Refs. $[9,46]$.

\footnotetext{
${ }^{3}$ Here we neglect the $Z$ boson exchange diagrams since their entire contribution to the total cross section is less than $1 \%$ from our numerical studies.
} 
This paper is organized as follows. In Sec. II, we present the polarized differential cross section of $e^{+} e^{-} \rightarrow Z_{d \gamma}$ for different $Z_{d}$ polarizations. For $\sqrt{s} \gg m_{Z_{d}}$, we show that the production of longitudinal $Z_{d}$ is suppressed due to WardTakahashi identity, i.e., $Z_{d}$ is transversely polarized in such a limit. In Sec. III, we discuss the method for probing the parity violation parameter $\rho$ in $e^{+} e^{-}$colliders. We present angular distributions of leptons arising from polarized $Z_{d}$ decays. Combining with angular distributions of $Z_{d}$ in production process, we construct the double angular distribution for the signal process $e^{+} e^{-} \rightarrow \gamma Z_{d} \rightarrow \gamma l^{+} l^{-}$. It will be shown that this double angular distribution depends on $\rho^{2}$ rather than $\rho$. We bin the signal events according to the sign of $J \equiv \cos \theta \times \cos \theta_{d}$ where $\theta$ is the angle of $Z_{d}$ with respect to the $e^{-}$direction in $e^{+} e^{-}$center of momentum $(\mathrm{CM})$ frame while $\theta_{d}$ is the helicity angle of lepton arising from $Z_{d}$ decay. The asymmetry $\mathcal{A}_{\mathrm{PN}} \equiv(S(J>0)-$ $S(J<0)) /(S(J>0)+S(J<0))$ with $S$ the number of signal events will be shown to be proportional to $\rho^{2}$, so that it directly reflects the degree of parity violation. In Sec. IV, event numbers of $e^{+} e^{-} \rightarrow \gamma \mu^{+} \mu^{-}$from signal and background are calculated with specific integrated luminosity in Belle II detector, taking into account the detector acceptance and energy resolutions. We also calculate the asymmetry parameter $\mathcal{A}_{\mathrm{PN}}$ which depends on the detector acceptance. It will be shown that the simultaneous fitting to $J>0$ and $J<0$ event bins should improve the significance of dark boson detection from simply counting the total event excess. The degree of improvement is closely related to $\mathcal{A}_{\mathrm{PN}}$. In addition, it is possible to measure $\mathcal{A}_{\mathrm{PN}}$ in Belle II detector. We estimate the statistical errors for such measurements under Belle II design integrated luminosity. We summarize and conclude in Sec. V.

\section{THE POLARIZED DARK BOSON PRODUCTION CROSS SECTION}

\section{A. Ward-Takahashi identity and the polarization of $Z_{d}$}

Let us write the amplitude for $e^{-}\left(p_{1}\right)+e^{+}\left(p_{2}\right) \rightarrow$ $Z_{d}\left(k_{1}\right)+\gamma\left(k_{2}\right)$ as $\mathcal{M} \equiv \mathcal{M}_{\mu} \epsilon^{\mu}\left(k_{1}\right)$ with $\epsilon^{\mu}\left(k_{1}\right)$ the polarization vector of $Z_{d}$. Here $\mathcal{M}_{\mu}$ contains the photon polarization vector. In the case that $Z_{d}$ is longitudinal, one has $\epsilon^{\mu}\left(k_{1}\right)=\left(\left|\vec{k}_{1}\right|, E_{Z_{d}} \hat{k}_{1}\right) / m_{Z_{d}}$. In the limit that $\sqrt{s} \gg m_{Z_{d}}$, i.e., $Z_{d}$ is ultrarelativistic, one has $\epsilon_{Z_{d}}^{\mu}=k_{1}^{\mu} / m_{Z_{d}}+$ $\mathcal{O}\left(m_{Z_{d}} / E_{Z_{d}}\right)$. Hence, $\mathcal{M}=\mathcal{M}_{\mu} k_{1}^{\mu} / m_{Z_{d}}+\mathcal{O}\left(m_{Z_{d}} / E_{Z_{d}}\right)$. However, $\mathcal{M}_{\mu} k_{1}^{\mu}=0$ in the limit $m_{e} \rightarrow 0$ as implied by Ward-Takahashi identity [30]. Therefore, the amplitude for a longitudinal polarized $Z_{d}$ is of the order $m_{Z_{d}} / \sqrt{s}$.

\section{B. Explicit demonstration of $\mathcal{M}_{\|}$suppression}

The square of $e^{-}\left(p_{1}\right)+e^{+}\left(p_{2}\right) \rightarrow Z_{d}\left(k_{1}\right)+\gamma\left(k_{2}\right)$ amplitude for a given $Z_{d}$ polarization can be expressed as follows:

$$
\begin{aligned}
\frac{|\overline{\mathcal{M}}|^{2}}{4}= & 16 \pi^{2} \alpha^{2} \varepsilon^{2}\left(g_{f, V}^{2}+g_{f, A}^{2}\right)\left[\frac{u}{t}+\frac{t}{u}+\frac{2 m_{Z_{d}}^{2}}{t u}\left(s-2\left(p_{1} \cdot \epsilon^{*}\right)\left(p_{1} \cdot \epsilon\right)-2\left(p_{2} \cdot \epsilon^{*}\right)\left(p_{2} \cdot \epsilon\right)\right)\right] \\
& -64 i \pi^{2} \alpha^{2} \varepsilon^{2} g_{f, V} \cdot g_{f, A} \times\left(\frac{1}{t}\right) \times \epsilon^{\rho \beta \sigma \nu} p_{1, \rho}\left(p_{2, \sigma}-k_{1, \sigma}\right) \epsilon_{\beta} \epsilon_{\nu}^{*} \\
& +64 i \pi^{2} \alpha^{2} \varepsilon^{2} g_{f, V} \cdot g_{f, A} \times\left(\frac{1}{u}\right) \times \epsilon^{\rho \beta \sigma \nu} p_{2, \rho}\left(p_{1, \sigma}-k_{1, \sigma}\right) \epsilon_{\beta} \epsilon_{\nu}^{*} \\
& -128 i \pi^{2} \alpha^{2} \varepsilon^{2} g_{f, V} \cdot g_{f, A} \times\left(\frac{1}{t u}\right) \times p_{2, \sigma} p_{1, \lambda} k_{1, \rho} \times \epsilon^{\rho \sigma \lambda \nu}\left(\left(p_{2} \cdot \epsilon\right) \epsilon_{\nu}^{*}+\left(p_{2} \cdot \epsilon^{*}\right) \epsilon_{\nu}\right) \\
& +128 i \pi^{2} \alpha^{2} \varepsilon^{2} g_{f, V} \cdot g_{f, A} \times\left(\frac{1}{t u}\right) \times p_{2, \sigma} p_{1, \lambda} k_{1, \rho} \times \epsilon^{\rho \sigma \lambda \nu}\left(\left(p_{1} \cdot \epsilon\right) \epsilon_{\nu}^{*}+\left(p_{1} \cdot \epsilon^{*}\right) \epsilon_{\nu}\right),
\end{aligned}
$$

where $\overline{\mathcal{M}}$ is the amplitude with the polarizations of initial fermions and final-state photon summed, $\alpha$ is the fine-structure constant, $m_{Z_{d}}$ and $\epsilon_{\mu}$ are dark boson mass and polarization vector, respectively, $s, t$, and $u$ are Mandelstam variables. It is clear that those terms proportional to $g_{f, V} \cdot g_{f, A}$ vanish by summing the $Z_{d}$ polarization, $\sum_{\lambda} \epsilon_{\mu}^{\lambda} \epsilon_{\nu}^{* \lambda}=-g_{\mu \nu}+k_{1, \mu} k_{1, \nu} / m_{Z_{d}}^{2}$. In the CM frame of colliding electrons and positrons, the momenta of initial and final-state particles are given by

$$
\begin{aligned}
p_{1}^{\mu} & =(E, 0,0,+E), \\
p_{2}^{\mu} & =(E, 0,0,-E), \\
k_{1}^{\mu} & =\left(E_{Z_{d}}, \omega \sin \theta, 0, \omega \cos \theta\right), \\
k_{2}^{\mu} & =(\omega,-\omega \sin \theta, 0,-\omega \cos \theta),
\end{aligned}
$$

where $\omega$ is the photon energy, $E_{Z_{d}}=\sqrt{\omega^{2}+m_{Z_{d}}^{2}}$, and $s=\left(p_{1}+p_{2}\right)^{2}=4 E^{2}$. Using energy and momentum 
conservation, we have $E_{Z_{d}}=E+m_{Z_{d}}^{2} /(2 \sqrt{s})$ and $\omega=E-m_{Z_{d}}^{2} /(2 \sqrt{s})$. Let us denote the amplitude for each polarization as $\mathcal{M}_{+}, \mathcal{M}_{-}$, and $\mathcal{M}_{\|}$for right-handed, lefthanded, and longitudinal polarized dark boson final state, respectively. We have

$$
\begin{aligned}
|\overline{\mathcal{M}}|_{+}^{2}= & \frac{8 \pi^{2} \alpha^{2} \varepsilon^{2}}{\left(t-m_{e}^{2}\right)\left(u-m_{e}^{2}\right)} \\
& \times\left[\left(1+\cos ^{2} \theta\right)\left(s^{2}+m_{Z_{d}}^{4}\right)+\rho \cos \theta\left(s-m_{Z_{d}}^{2}\right)^{2}\right], \\
|\overline{\mathcal{M}}|_{-}^{2}= & \frac{8 \pi^{2} \alpha^{2} \varepsilon^{2}}{\left(t-m_{e}^{2}\right)\left(u-m_{e}^{2}\right)} \\
& \times\left[\left(1+\cos ^{2} \theta\right)\left(s^{2}+m_{Z_{d}}^{4}\right)-\rho \cos \theta\left(s-m_{Z_{d}}^{2}\right)^{2}\right], \\
|\overline{\mathcal{M}}|_{\|}^{2}= & \frac{8 \pi^{2} \alpha^{2} \varepsilon^{2}}{\left(t-m_{e}^{2}\right)\left(u-m_{e}^{2}\right)}\left(4 m_{Z_{d}}^{2} \sin ^{2} \theta\right),
\end{aligned}
$$

where the normalization $g_{f, V}^{2}+g_{f, A}^{2}=1$ has been taken and $\rho=4 g_{f, V} g_{f, A}$. The absolute value of $\rho$ essentially describes the degree of parity violation. It is shown that $|\overline{\mathcal{M}}|_{\|}^{2}$ is suppressed by $m_{Z_{d}}^{2} / s$ compared to $|\overline{\mathcal{M}}|_{ \pm}^{2}$. In Fig. 1, we present the fraction of matrix element square as a function of $\cos \theta$ for each helicity state of $Z_{d}$. We take the $V-A$ case with $g_{f, V}=-g_{f, A}=1 / \sqrt{2}$ for illustration. The upper, middle, and lower panels correspond to $m_{Z_{d}} / \sqrt{s}=0.1,0.3$, and 0.8 , respectively. For the first two cases, one can see that the longitudinal fraction is no more than $10 \%$. In addition, $|\overline{\mathcal{M}}|_{-}^{2}$ dominates the forward direction $(0 \leq \cos \theta \leq 1)$, while $|\overline{\mathcal{M}}|_{+}^{2}$ dominates the backward direction. For the third case, the longitudinal fraction is non-negligible and the fractions for helicity +1 and -1 states are almost identical due to the suppression of $\rho$-dependent terms, i.e., the forward-backward asymmetry approaches to zero in the limit $s \rightarrow m_{Z_{d}}^{2}$.

The polarized differential cross section is readily calculated with

$$
\frac{d \sigma_{i}}{d \cos \theta}=\frac{1}{32 \pi s}\left(1-\frac{m_{Z_{d}}^{2}}{s}\right)|\mathcal{M}|_{i}^{2}
$$

with $i=+,-$, and $\|$. To check our result, we take $\varepsilon=1$ and sum over contributions from all polarizations. In the limit $s \gg m_{e}^{2}, m_{Z_{d}}^{2}$, our result approaches to the differential cross section of $e^{+} e^{-} \rightarrow \gamma \gamma$. The results for polarized differential cross section are shown in Fig. 2. In this calculation, we take $\varepsilon=7 \times 10^{-4}$ for $m_{Z_{d}} / \sqrt{s}=0.1$, 0.3 , and 0.8 , respectively, for illustrations. This $\varepsilon$ value is reachable by Belle II with $500 \mathrm{fb}^{-1}$ luminosity for $m_{Z_{d}}$ around $1 \mathrm{GeV}$ [31]. Similar to the case of amplitude square, the longitudinal polarized contribution is suppressed for small $m_{Z_{d}} / \sqrt{s}$. We present in Fig. 3 the fraction of
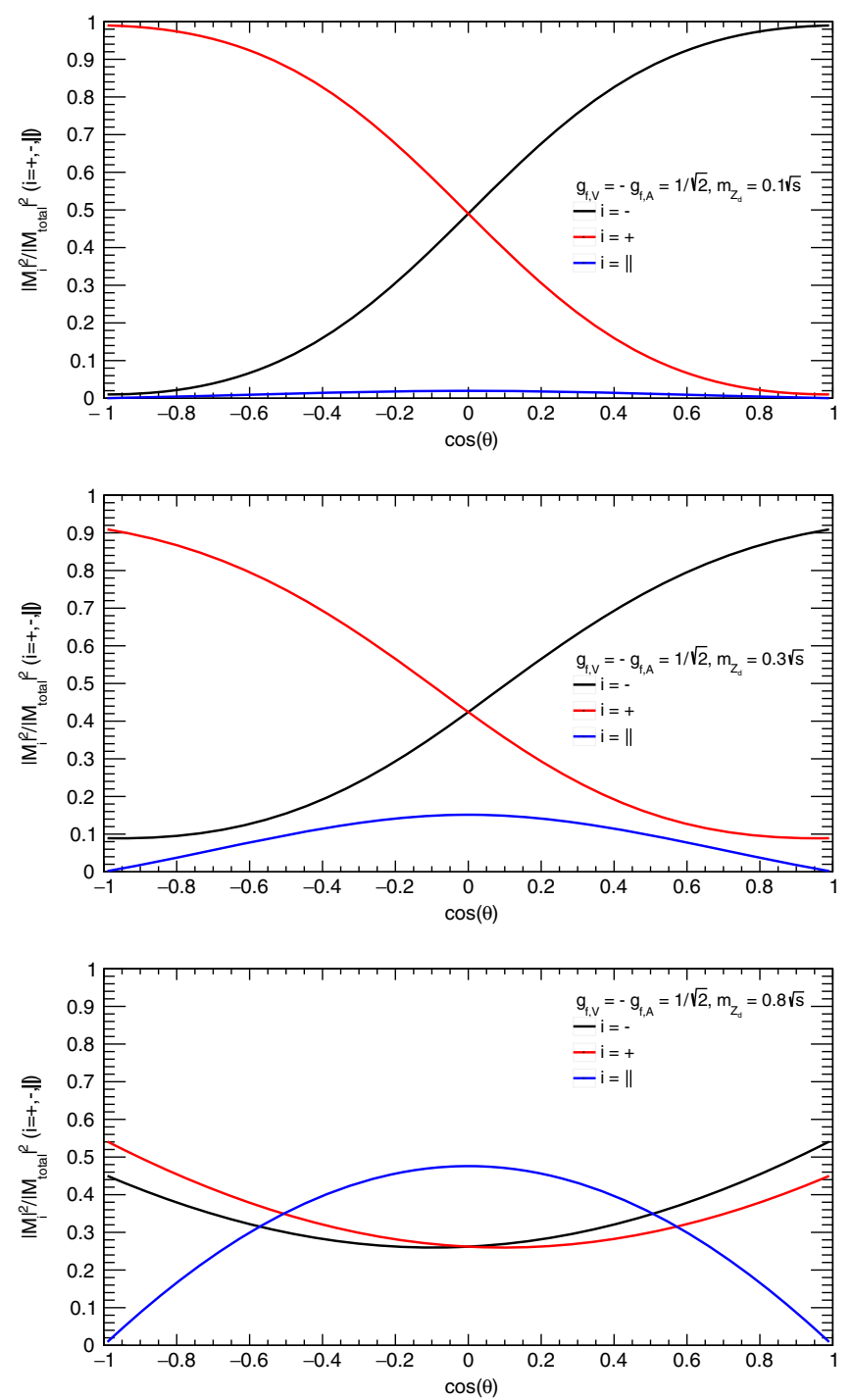

FIG. 1. The fraction of matrix element square for helicity +1 , -1 , and longitudinal dark boson final state. We take the $V-A$ case with $g_{f, V}=-g_{f, A}=1 / \sqrt{2}$. The ratio $m_{Z_{d}} / \sqrt{s}$ is taken to be $0.1,0.3$, and 0.8 on upper, middle, and lower panels, respectively.

differential cross section in each $Z_{d}$ polarization. Each fraction is represented by a region of specific color.

\section{PROBING PARITY VIOLATION EFFECTS IN $e^{+} e^{-} \rightarrow Z_{d} \gamma \rightarrow Z_{d} \mu^{+} \mu^{-}$}

Now we consider the angular distributions of $Z_{d}$ decays. Through mixing with SM gauge bosons, $Z_{d}$ can decay to SM leptons with the polarized differential decay rate

$$
\frac{d \Gamma_{l^{+} l^{-}}^{i}}{d \cos \theta_{d}}=\frac{\beta}{32 \pi m_{Z_{d}}}\left|\mathcal{M}\left(Z_{d}^{(i)} \rightarrow l^{+} l^{-}\right)\right|^{2},
$$

with $i=+,-$, and $\|, \beta=p_{l} / E_{l}=\sqrt{1-4 m_{l}^{2} / m_{Z_{d}}^{2}}$, and $\theta_{d}$ the angle between $l^{-}$direction in the $Z_{d}$ rest frame and the $Z_{d}$ direction in $e^{+} e^{-} \mathrm{CM}$ frame. Thus, we obtain 

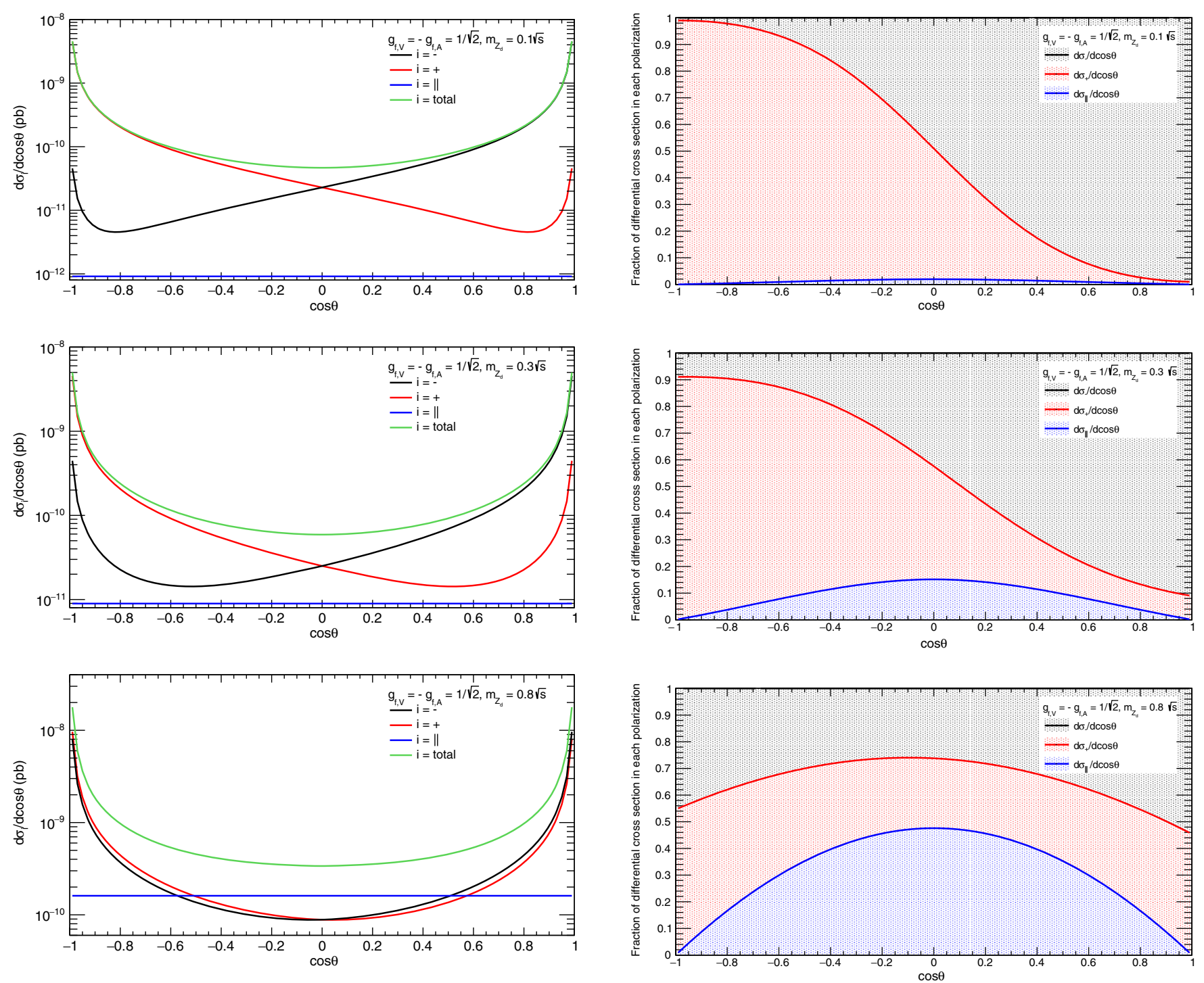

FIG. 2. The polarized differential cross section for $e^{+} e^{-} \rightarrow Z_{d} \gamma$ with $\varepsilon=7 \times 10^{-4}$ at Belle II for $m_{Z_{d}} / \sqrt{s}=0.1,0.3$, and 0.8 , respectively.

$\frac{d \Gamma_{l^{+} l^{-}}^{+}}{d \cos \theta_{d}}=\frac{\alpha \varepsilon^{2} \beta}{2 m_{Z_{d}}}\left[2 g_{l, V}^{2} m_{l}^{2}+\left(1+\cos ^{2} \theta_{d}\right) p_{l}^{2}+\rho \cos \theta_{d} E_{l} p_{l}\right]$,

FIG. 3. The fraction of differential cross section in each $Z_{d}$ polarization as represented by regions of different colors. The range for each color adds up to unity.

$$
\frac{d^{2} P}{d \kappa d \xi}=\frac{1}{\sigma_{T} \cdot \Gamma_{l^{+} l^{-}}} \sum_{i}\left(\frac{d \sigma^{i}}{d \cos \theta}\right) \cdot\left(\frac{d \Gamma_{l^{+} l^{-}}^{i}}{d \cos \theta_{d}}\right)
$$

$\frac{d \Gamma_{l^{+} l^{-}}^{-}}{d \cos \theta_{d}}=\frac{\alpha \varepsilon^{2} \beta}{2 m_{Z_{d}}}\left[2 g_{l, V}^{2} m_{l}^{2}+\left(1+\cos ^{2} \theta_{d}\right) p_{l}^{2}-\rho \cos \theta_{d} E_{l} p_{l}\right]$,

$\frac{d \Gamma_{l^{+} l^{-}}^{\|}}{d \cos \theta_{d}}=\frac{\alpha \varepsilon^{2} \beta}{m_{Z_{d}}}\left[g_{l, V}^{2} m_{l}^{2}+\sin ^{2} \theta_{d} p_{l}^{2}\right]$.

Given $g_{l, V}^{2}+g_{l, A}^{2}=1$ and $\rho=4 g_{l, V} g_{l, A}$, we have $g_{l, V}^{2}=$ $\left(1+\sqrt{1-\rho^{2} / 4}\right) / 2$ for $\left|g_{l, V}\right| \geq\left|g_{l, A}\right|$, while $g_{l, V}^{2}=$ $\left(1-\sqrt{1-\rho^{2} / 4}\right) / 2$ for $\left|g_{l, V}\right| \leq\left|g_{l, A}\right|$. The double differential distribution of final-state leptons is given by 
To analyze the double distribution, we integrate $\kappa$ from -1 to 0 , i.e., we consider leptonic decays of $Z_{d}$ produced in the backward direction. The forward-backward asymmetry of $l^{-}$in its helicity angle is presented in Fig. 4. In three panels, we present the angular distribution of $l^{-}$for $|\rho|=0$, 1 , and 2, respectively. The case $|\rho|=0$ implies either $g_{l, A}=0$ or $g_{l, V}=0$ with the former corresponding to the dark-photon scenario, while $|\rho|=2$ corresponds to either $V-A$ or $V+A$ cases. On the upper panel, we separate results into $\left|g_{l, V}\right| \geq\left|g_{l, A}\right|$ and $\left|g_{l, V}\right| \leq\left|g_{l, A}\right|$. These two cases coincide in the middle and lower panels with $\beta=1$, i.e., $m_{l} \rightarrow 0$. The asymmetries in the upper and lower panels are small either due to a small $\beta$ or to the suppression
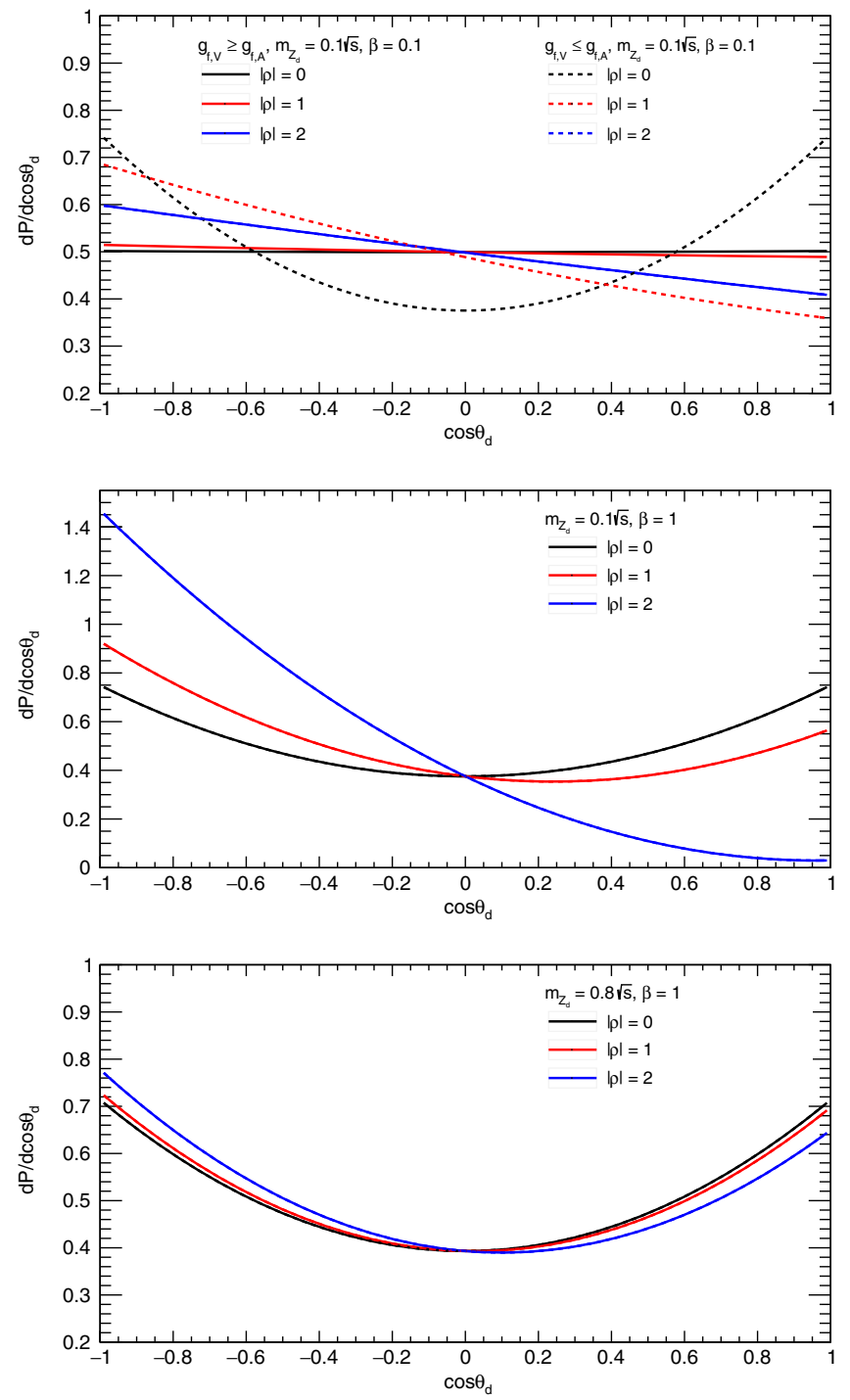

FIG. 4. The angular distribution of $l^{-}$from decays of $Z_{d}$ produced in the backward direction by $e^{+} e^{-} \rightarrow Z_{d} \gamma$ for $|\rho|=0$, 1 , and 2 , respectively. The upper panel is for relativistic $Z_{d}$ with $m_{Z_{d}}=0.1 \sqrt{s}$ and nonrelativistic lepton with $\beta=0.1$. The middle panel corresponds to $\left(\beta, m_{Z_{d}} / \sqrt{s}\right)=(1,0.1)$, while the lower panel corresponds to $\left(\beta, m_{Z_{d}} / \sqrt{s}\right)=(1,0.8)$. in $\left(1-m_{Z_{d}}^{2} / s\right)^{2}$ with $m_{Z_{d}} / \sqrt{s}=0.8$. Significant asymmetry is seen in the middle panel with large $|\rho|$. In general, we may define the following asymmetry parameter:

$$
\mathcal{A}_{\mathrm{PN}} \equiv \frac{S(\kappa \cdot \xi>0)-S(\kappa \cdot \xi<0)}{S(\kappa \cdot \xi<0)+S(\kappa \cdot \xi>0)}
$$

where the subscript PN indicates that $\mathcal{A}_{\mathrm{PN}}$ describes the difference in signal event rate as $\kappa \xi$ reverses its sign. In limits of $\beta \rightarrow 1$ and $m_{Z_{d}} \ll \sqrt{s}$, we have

$$
\mathcal{A}_{\mathrm{PN}}=\frac{3}{4}\left(\frac{\rho^{2}}{4}\right) \frac{-\ln \left(1-\kappa_{m}^{2}\right)}{\ln \left(\frac{1+\kappa_{m}}{1-\kappa_{m}}\right)-\kappa_{m}},
$$

where $\kappa_{m}$ is the maximum of $\kappa$. The minimum of $\kappa$ is assumed to be $-\kappa_{m}$. It is found that $\mathcal{A}_{\mathrm{PN}}$ is not very sensitive to $\kappa_{m}$. $\mathcal{A}_{\mathrm{PN}}=0.64 \times\left(\rho^{2} / 4\right)$ for $\kappa_{m}=0.95$, and $0.55 \times\left(\rho^{2} / 4\right)$ for $\kappa_{m}=0.80$. In the next section, we shall calculate $\mathcal{A}_{\mathrm{PN}}$ and its associated uncertainty with acceptance cuts in Belle II detector.

\section{THE PROSPECT OF MEASURING PARITY-VIOLATING DARK BOSON INTERACTIONS IN BELLE II}

In this section, we discuss the search for dark vector boson and the possible measurement of parity violation parameter $\rho$ through determining $\mathcal{A}_{\mathrm{PN}}$ in Belle II detector. We shall begin by considering the detector acceptance of Belle II and compare our sensitivity estimation for the dark photon search through $e^{+} e^{-} \rightarrow A^{\prime} \gamma$ with $A^{\prime} \rightarrow \mu^{+} \mu^{-}$with $B A B A R$ result at $514 \mathrm{fb}^{-1}$ and the projected sensitivity of Belle II at $500 \mathrm{fb}^{-1}$. These comparisons are important for validating our approach. Next, we consider Belle II at the full integrated luminosity $50 \mathrm{ab}^{-1}$ and extend our discussions to the dark boson scenario with a nonvanishing $\rho$.

\section{A. Sensitivity for the dark photon search at Belle II}

To illustrate our points in previous sections, we take the dark photon mass as 0.5 and $2 \mathrm{GeV}$, respectively, as benchmark values. These two mass values satisfy $m_{Z_{d}} / \sqrt{s} \ll 1$ so that the dark photons are produced in transversely polarized states. Hence, for the generalization to dark boson scenario in the next subsection, we shall see that the asymmetry parameter $\mathcal{A}_{\mathrm{PN}}$ will be significant.

Let us begin by taking $m_{Z_{d}}=0.5 \mathrm{GeV}$ with $\rho=0$. In this case, the branching ratio for $Z_{d} \rightarrow \mu^{+} \mu^{-}$is about $40 \%$ from the measurement of $R=\sigma\left(e^{+} e^{-} \rightarrow\right.$ hadrons $) /$ $\sigma\left(e^{+} e^{-} \rightarrow \mu^{+} \mu^{-}\right)[14,47]$. The Belle II calorimeter angular coverage is $12.4^{\circ} \leq \theta_{\gamma}^{\text {lab }} \leq 155.1^{\circ}$ [48], which detects finalstate photon in the rapidity range $-1.51 \leq \eta_{\gamma}^{\text {lab }} \leq 2.22$. Since the boost velocity from the laboratory frame to $\mathrm{CM}$ frame is $\beta_{\mathrm{CM}}=\left(E_{e^{-}}-E_{e^{+}}\right) /\left(E_{e^{-}}+E_{e^{+}}\right)=3 / 11$, the photon rapidity in the $\mathrm{CM}$ frame is given by 
$\eta_{\gamma}^{\mathrm{CM}}=\eta_{\gamma}^{\mathrm{lab}}+\ln \left(\left(1-\beta_{\mathrm{CM}}\right) /\left(1+\beta_{\mathrm{CM}}\right)\right) / 2$. Hence, $-1.79 \leq$ $\eta_{\gamma}^{\mathrm{CM}} \leq 1.94$. Furthermore, the angular coverage of $K_{L^{-}}$ Muon detector [48] is $25^{\circ} \leq \theta_{\mu^{ \pm}}^{\text {lab }} \leq 150^{\circ}$. This leads to the muon rapidity range $-1.60 \leq \eta_{\mu^{ \pm}}^{\mathrm{CM}} \leq 1.23$ in the $\mathrm{CM}$ frame. Since the signal mass resolution is between 1.5 and $8 \mathrm{MeV}$ in $B A B A R$ analysis [24], we take it to be $5 \mathrm{MeV}$ for our sensitivity estimation, i.e., the signal and background events are calculated within a $5 \mathrm{MeV} \mu^{+} \mu^{-}$invariant mass window in the vicinity of assumed $Z_{d}$ mass.

We note that the rapidity cuts preserve $S(\kappa \cdot \xi>0)=$ $S(\kappa \cdot \xi<0)$ for $\rho=0$. Using CalcHEP [49], we find that the signal $e^{+} e^{-} \rightarrow \gamma Z_{d}$ with $Z_{d} \rightarrow \mu^{+} \mu^{-}$has the cross section $1.84 \times 10^{3} \cdot \varepsilon^{2}$ pb by considering both the rapidity cuts and the $40 \% Z_{d} \rightarrow \mu^{+} \mu^{-}$branching ratio, and the cross section for QED background process $e^{+} e^{-} \rightarrow \gamma \mu^{+} \mu^{-}$with the same acceptance cut is $7.76 \times 10^{-2} \mathrm{pb}$. We note that the above parametrization for signal cross section is valid only for $\varepsilon<0.3$ such that the $\varepsilon$-dependent $Z_{d}$ width is less than $10 \%$ of the signal mass resolution. With $500 \mathrm{fb}^{-1}$ of integrated luminosity, the Belle II $90 \%$ C.L. sensitivity to $\varepsilon$ is estimated by the following $\chi^{2}$ function:

$$
\chi^{2}=2\left(n \ln \left(\frac{n}{w}\right)+w-n\right),
$$

where $n$ is the observed event number while $w$ is the expected event number. With $n=S+B=$ $\left(1.84 \times 10^{3} \cdot \varepsilon^{2}+7.76 \times 10^{-2}\right) \mathrm{pb} \cdot 500 \mathrm{fb}^{-1}, \quad w=B=$ $7.76 \times 10^{-2} \mathrm{pb} \cdot 500 \mathrm{fb}^{-1}$, and $\chi^{2}=(1.645)^{2}$ for $90 \%$ C.L. sensitivity, ${ }^{4}$ we obtain $\varepsilon=5.9 \times 10^{-4}$, which is consistent with the sensitivity $\varepsilon=5.6 \times 10^{-4}$ given in Belle II physics book for the visible modes $Z_{d} \rightarrow e^{+} e^{-}, \mu^{+} \mu^{-}$[31]. The latter is also comparable to the constraint from $B A B A R$ search via visible modes at $514 \mathrm{fb}^{-1}$ [24].

We next take $m_{Z_{d}}=2 \mathrm{GeV}$ with $\rho=0$. In this case, the branching ratio of $Z_{d} \rightarrow \mu^{+} \mu^{-}$is about $24 \%$ [47]. Hence, the signal cross section is around $1.11 \times 10^{3} \cdot \varepsilon^{2} \mathrm{pb}$, while the background cross section is $2.54 \times 10^{-2} \mathrm{pb}$. Following Eq. (13), we obtain Belle II $90 \%$ C.L. sensitivity to $\varepsilon$ as $\varepsilon=5.8 \times 10^{-4}$, which is also not much different from $6.6 \times 10^{-4}$ given by Belle II physics book for the visible modes $Z_{d} \rightarrow e^{+} e^{-}, \mu^{+} \mu^{-}$[31].

\section{B. Probing the parity violation effects}

\section{Enhancement on the detection significance}

In the case of nonvanishing $\rho$, we modify the $\chi^{2}$ function in Eq. (13) into

\footnotetext{
${ }^{4}$ In principle, one should also consider the interference between signal and background amplitudes for calculating $S+B$. On the other hand, it can be shown that such contribution scales as $\varepsilon^{2}$, and for the current case the interference part of the cross section is $\sim-1.8 \cdot \varepsilon^{2} \mathrm{pb}$, which is negligible.
}

$\chi^{2}=2\left(n_{a} \ln \left(\frac{n_{a}}{w_{a}}\right)+w_{a}-n_{a}\right)+2\left(n_{b} \ln \left(\frac{n_{b}}{w_{b}}\right)+w_{b}-n_{b}\right)$,

where $n_{a}\left(w_{a}\right)$ and $n_{b}\left(w_{b}\right)$ are observed (expected) event numbers in $\kappa \cdot \xi>0$ and $\kappa \cdot \xi<0$ bins, respectively. By considering separate event bins, the dark boson detection significance is expected to be improved. With $n_{a, b}=S_{a, b}+B_{a, b}$ and $w_{a, b}=B_{a, b}$, we can show that

$$
\chi^{2}=\frac{S^{2}}{B}\left(1+\mathcal{A}_{\mathrm{PN}}^{2}\right),
$$

with the assumption $S_{a, b} \ll B_{a, b}$ and the identity $B_{a}=B_{b}=B / 2$. We note that $S_{a}$ and $S_{b}$ are the event excess in $\kappa \cdot \xi>0$ and $\kappa \cdot \xi<0$ event bins, respectively. It is clear that the detection significance increases from $[S / \sqrt{B}] \cdot \sigma$ to $\left[S \sqrt{\left(1+\mathcal{A}_{\mathrm{PN}}^{2}\right)} / \sqrt{B}\right] \cdot \sigma$ by considering separate event bins.

\section{Measurement of $\mathcal{A}_{\mathrm{PN}}$}

Following Eq. (11), we have $\mathcal{A}_{\mathrm{PN}}=\left(S_{a}-S_{b}\right) /\left(S_{a}+\right.$ $S_{b}$ ) with the statistical uncertainties of both numerator and denominator being $\sqrt{B}$. Hence, the uncertainty of $\mathcal{A}_{\mathrm{PN}}$ is given by $\sigma_{\mathcal{A}_{\mathrm{PN}}}=\sqrt{1+\mathcal{A}_{\mathrm{PN}}^{2}}(\sqrt{B} / S)$. We note that the asymmetry parameter $\mathcal{A}_{\mathrm{PN}}$ as defined by Eq. (11) is actually independent of the integrated luminosity. It can be calculated by the scattering cross section with appropriate kinematic cuts imposed.

\section{Numerical results}

Let us assume the scenario that one achieves a 5 standard deviation detection of dark boson at the designed integrated luminosity $50 \mathrm{ab}^{-1}$ based upon counting the overall event excess, i.e., $S=5 \sqrt{B}$ according to Eq. (13). On the other hand, the event excess $S_{a}$ and $S_{b}$ for each event bin depends on the asymmetry parameter $\mathcal{A}_{\mathrm{PN}}$. It is to be noted that we shall fix the total event excess $S \equiv S_{a}+S_{b}$ regardless of the $\rho$ value. Hence, $\sigma\left(e^{+} e^{-} \rightarrow Z_{d} \gamma\right) \cdot \operatorname{Br}\left(Z_{d} \rightarrow \mu^{+} \mu^{-}\right)$is fixed. Since $\sigma\left(e^{+} e^{-} \rightarrow Z_{d} \gamma\right) \propto \varepsilon^{2}$ while $\operatorname{Br}\left(Z_{d} \rightarrow \mu^{+} \mu^{-}\right)$ depends on $\rho$, the $\varepsilon$ value extracted from $S=5 \sqrt{B}$ at $50 \mathrm{ab}^{-1}$ also depends on $\rho$.

To proceed with our numerical analysis, we first calculate $\mathcal{A}_{\mathrm{PN}}$ and its uncertainty as a function of the ratio $m_{Z_{d}} / \sqrt{s}$ for a given $\rho$. In Fig. 5, we present results for $\rho=0.5,1.0,1.5$, and 2.0 , respectively. The results for other $\rho$ 's can be easily inferred since $\mathcal{A}_{\mathrm{PN}} \propto \rho^{2}$. It is seen that $\mathcal{A}_{\mathrm{PN}}$ is consistent with zero for $\rho=0.5$ and $\rho=1$, since the $1 \sigma$ error bars in these cases reach $\mathcal{A}_{\mathrm{PN}}<0$ regime. On the other hand, for $\rho=2, \mathcal{A}_{\mathrm{PN}}$ are nonvanishing at more than $2 \sigma$ for $m_{Z_{d}} / \sqrt{s} \leq 0.3$. With $\mathcal{A}_{\mathrm{PN}}$ determined, the enhancement on detection significance according to Eq. (15) can be calculated. Let us consider two special scenarios for $\rho$. The 

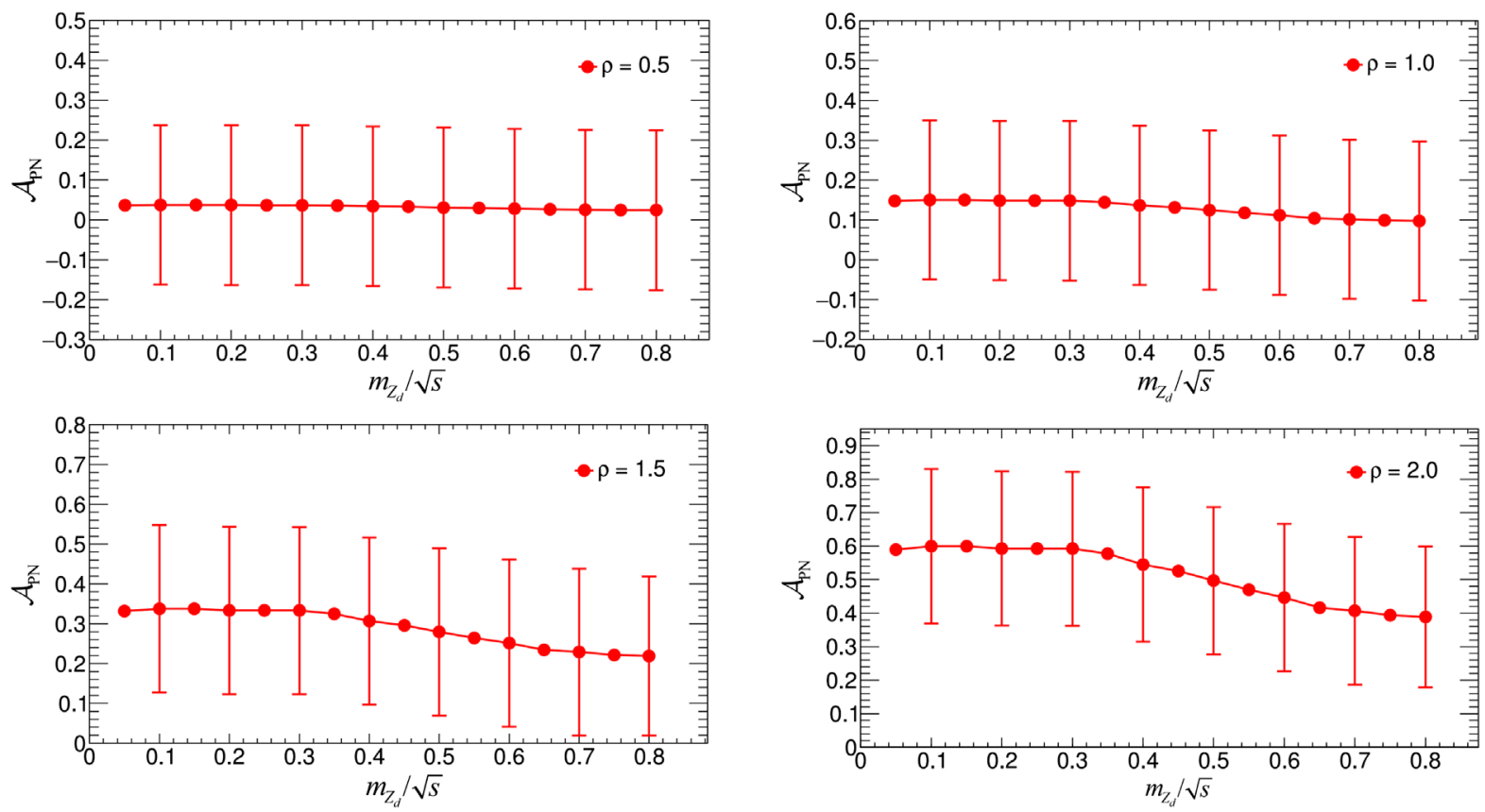

FIG. 5. $\mathcal{A}_{\mathrm{PN}}$ and its uncertainty as functions of $m_{Z_{d}} / \sqrt{s}$ for $S / \sqrt{B}=5$. We present results for $\rho=0.5,1.0,1.5$, and 2.0, respectively.

first scenario is setting $\varepsilon_{\gamma}=\varepsilon_{Z}$ in Eq. (2). Recasting the coupling of $Z_{d}$ to leptons into the standard form $e \bar{l}\left(g_{l, V} \gamma_{\mu}+g_{l, A} \gamma_{\mu} \gamma_{5}\right) l Z_{d}^{\mu}$, we have $\varepsilon=1.18 \varepsilon_{\gamma}$, $g_{l, V}=-0.87$, and $g_{l, A}=-0.5$, which leads to $\rho=1.74$. The second scenario is the extreme asymmetry case, such as $V-A$ interaction between $Z_{d}$ and SM fermions. This case is achieved with $\varepsilon_{\gamma}=\varepsilon_{Z} \tan \theta_{W}$ such that $\varepsilon=0.83 \varepsilon_{Z}$ and $g_{l, A}=-g_{l, V}=1 / \sqrt{2}$, which leads to $\rho=-2$. In this case, the dark boson interacts with the up-type (down-type) quark the same way as it interacts with neutrino (charged lepton). The values of $\mathcal{A}_{\mathrm{PN}}$ and the associated uncertainties in these two scenarios are summarized in Table I for $m_{Z_{d}}=0.5$ and $2 \mathrm{GeV}$. The improved detection significance with Eq. (15) is also presented there. We note that, for $|\rho|=1.74$, the significance for a nonvanishing $\mathcal{A}_{\mathrm{PN}}$ already reaches $2 \sigma$.

It is of interest to estimate $S$ for a $5 \sigma$ detection at $50 \mathrm{ab}^{-1}$. Given the background cross sections $7.76 \times 10^{-2}$ and
$2.54 \times 10^{-2} \mathrm{pb}$ for $m_{Z_{d}}=0.5$ and $2 \mathrm{GeV}$, respectively, we have $B=3.88 \times 10^{6}$ and $1.27 \times 10^{6}$, respectively. For $S=5 \sqrt{B}$ assumed here, $S=9850$ and 5634 for $m_{Z_{d}}=0.5$ and $2 \mathrm{GeV}$, respectively. The way $S$ is split into $S_{a}$ and $S_{b}$ depends on $\mathcal{A}_{\mathrm{PN}}$. We present values of $S_{a}$ and $S_{b}$ for benchmark values of $m_{Z_{d}}$ and $\rho$ in Table I. Finally, in the last two rows of Table I, we also present $\operatorname{Br}\left(Z_{d} \rightarrow \mu^{+} \mu^{-}\right)$ and the corresponding $\varepsilon$ extracted from $S=5 \sqrt{B}$ at $50 \mathrm{ab}^{-1}$.

Before closing this section, we comment on $Z_{d} \rightarrow \mu^{+} \mu^{-}$ branching ratio for a nonvanishing $\rho$. It is clear that $\operatorname{Br}\left(Z_{d} \rightarrow \mu^{+} \mu^{-}\right)$in the dark boson scenario differs from that of the dark photon case, in particular the decay $Z_{d} \rightarrow$ $\nu \bar{\nu}$ is possible for the former case. From Eq. (2), one obtains $Z_{d}$ couplings to leptons and quarks. For $|\rho|=1.74$ and $m_{Z_{d}}=0.5 \mathrm{GeV}$, we have $\operatorname{Br}\left(Z_{d} \rightarrow e^{+} e^{-}\right)=22 \%$, $\operatorname{Br}\left(Z_{d} \rightarrow \mu^{+} \mu^{-}\right)=21 \%, \quad \sum_{l} \operatorname{Br}\left(Z_{d} \rightarrow \nu_{l} \bar{\nu}_{l}\right)=33 \%$, and

TABLE I. Presented are the asymmetry parameter $\mathcal{A}_{\mathrm{PN}}$, the improved detection significance with two event bins, the signal event numbers, and extracted $\varepsilon$ values assuming $5 \sigma$ detection significance at $50 \mathrm{ab}^{-1}$ based upon overall event excess, i.e., $S=5 \sqrt{B}$, for benchmark $m_{Z_{d}}$ and $\rho$ values. The $Z_{d} \rightarrow \mu^{+} \mu^{-}$branching ratios are also presented.

\begin{tabular}{|c|c|c|c|c|c|c|}
\hline \multirow{2}{*}{$\begin{array}{l}|\rho| \\
m_{Z_{d}} / \mathrm{GeV}\end{array}$} & \multicolumn{2}{|c|}{0.00} & \multicolumn{2}{|c|}{1.74} & \multicolumn{2}{|c|}{2.00} \\
\hline & 0.5 & 2.0 & 0.5 & 2.0 & 0.5 & 2.0 \\
\hline $\mathcal{A}_{\mathrm{PN}}$ & $(0.0 \pm 0.2)$ & $(0.0 \pm 0.2)$ & $(0.43 \pm 0.22)$ & $(0.44 \pm 0.22)$ & $(0.58 \pm 0.23)$ & $(0.60 \pm 0.23)$ \\
\hline Det. Sig. (Eq. (15)) & $5.0 \sigma$ & $5.0 \sigma$ & $5.4 \sigma$ & $5.5 \sigma$ & $5.8 \sigma$ & $5.8 \sigma$ \\
\hline$S(\kappa \cdot \xi>0)$ & 4925 & 2817 & 7040 & 4053 & 7780 & 4507 \\
\hline$S(\kappa \cdot \xi<0)$ & 4925 & 2817 & 2810 & 1581 & 2070 & 1127 \\
\hline $\operatorname{Br}\left(Z_{d} \rightarrow \mu^{+} \mu^{-}\right)$ & $40 \%$ & $24 \%$ & $21 \%$ & $7.5 \%$ & $17 \%$ & $6.7 \%$ \\
\hline$\varepsilon \cdot 10^{4}$ & 3.3 & 3.2 & 4.6 & 5.7 & 5.1 & 6.1 \\
\hline
\end{tabular}


$\operatorname{Br}\left(Z_{d} \rightarrow\right.$ hadrons $)=24 \%$. For $m_{Z_{d}}=2 \mathrm{GeV}$ with the same $|\rho|$, the hadronic branching ratio increases to about $74 \%$ due to the opening of $Z_{d} \rightarrow s \bar{s}$ channel. Furthermore, $\sum_{l} \operatorname{Br}\left(Z_{d} \rightarrow \nu_{l} \bar{\nu}_{l}\right)=11 \%$ and $\operatorname{Br}\left(Z_{d} \rightarrow e^{+} e^{-}\right) \simeq$ $\operatorname{Br}\left(Z_{d} \rightarrow \mu^{+} \mu^{-}\right)=7.5 \%$. For $|\rho|=2, \operatorname{Br}\left(Z_{d} \rightarrow \mu^{+} \mu^{-}\right)=$ $17 \%$ and $6.7 \%$ for $m_{Z_{d}}=0.5$ and $2 \mathrm{GeV}$, respectively. We remark that our estimation of $\operatorname{Br}\left(Z_{d} \rightarrow\right.$ hadrons $)$ depends on the $Z_{d}$ mass. For $m_{Z_{d}}=2 \mathrm{GeV}$, we use the quark level process $Z_{d} \rightarrow q \bar{q}$ to calculate the hadronic decay width. For $m_{Z_{d}}=0.5 \mathrm{GeV}$, we consider only vector current contribution that can be inferred from the data as mentioned earlier. The axial-vector current is essentially not contributing since it cannot lead to two-pion final state due to parity conservation of strong interaction, nor its coupling to three-pion final state is noticeable at $0.5 \mathrm{GeV}$ from the study of axial-vector spectral function of hadronic $\tau$ decays [50].

\section{SUMMARY AND CONCLUSION}

In this paper, we have pointed out that the dark boson produced by $e^{+} e^{-} \rightarrow Z_{d} \gamma$ is transversely polarized in the limit $m_{Z_{d}} \ll \sqrt{s}$. This is a direct consequence of WardTakahashi identity. We also demonstrated this property by explicit calculations. The suppressed production of longitudinally polarized dark boson state is shown in Fig. 1 for the $V-A$ limit, i.e., $g_{l, V}=-g_{l, A}=1 / \sqrt{2}$. For $m_{Z_{d}} \ll \sqrt{s}$, the negative-helicity dark boson dominates the forward region $(\cos \theta>0)$, while the positive-helicity one dominates the backward region $(\cos \theta<0)$. As $m_{Z_{d}}$ approaches to $\sqrt{s}$, the production of longitudinally polarized dark boson becomes noticeable. Furthermore, the angular distributions of negative- and positive-helicity dark bosons become indistinguishable.

Since we aim for determining the parity violation parameter $\rho$, we analyze $\mu^{-}\left(\mu^{+}\right)$angular distributions from polarized $Z_{d}$ decays. The double distribution of final state muons $d^{2} P / d \kappa d \xi\left(\kappa=\cos \theta, \xi=\cos \theta_{d}\right)$, defined in Eq. (10), was shown to be sensitive to $\rho$. Explicitly, we found that $d^{2} P / d \kappa d \xi=Q_{0}+Q_{2} \rho^{2}$ with $Q_{0}$ an even function of both $\kappa$ and $\xi$ and $Q_{2}$ an odd function of these variables. This implies that the signal event number in the kinematic range $\kappa \cdot \xi>0$ differs from that with $\kappa \cdot \xi<0$, which motivates our definition of asymmetry parameter $\mathcal{A}_{\mathrm{PN}}$ proportional to $\rho^{2}$. Besides depending on $\rho^{2}, \mathcal{A}_{\mathrm{PN}}$ also depends on the detector acceptance. We calculate numbers of signature and background events for two benchmark masses $m_{Z_{d}}=0.5$ and $2 \mathrm{GeV}$ in Belle II detector. The resulting $90 \%$ C.L. sensitivity to $\varepsilon$ at $500 \mathrm{fb}^{-1}$ integrated luminosity is found to be consistent with that in Belle II physics book for the dark photon scenario.

In the general scenario with nonvanishing $\rho$, we have seen that the detection significance of dark bosons increases by separately considering events with different signs of $\kappa \cdot \xi$ rather than just counting the overall event excess. The increased $\chi^{2}$ value is proportional to $\mathcal{A}_{\mathrm{PN}}^{2}$, as seen from Eq. (15). The numerical values of $\mathcal{A}_{\mathrm{PN}}$ are calculated as a function of $m_{Z_{d}} / \sqrt{s}$ for $\rho=0.5,1.0,1.5$, and 2.0, respectively.

In conclusion, we have shown that the detection of dark boson decays into muon pairs in $e^{+} e^{-}$colliders can probe the parity-violating couplings between the dark boson and SM fermions. Assuming a $5 \sigma$ event excess in the search for $e^{+} e^{-} \rightarrow \gamma Z_{d}$ with $Z_{d} \rightarrow \mu^{+} \mu^{-}$at Belle II, we have seen that the simultaneous fitting to event numbers in positive and negative $\kappa \cdot \xi$ bins should improve the detection significance to $5.4 \sigma$ and $5.8 \sigma$ for input true models with $|\rho|=$ 1.74 and $|\rho|=2.0$, respectively. We have also seen that the significance for a nonvanishing $\mathcal{A}_{\mathrm{PN}}$ can reach $2 \sigma$ for $\varepsilon_{\gamma}=\varepsilon_{Z}(\rho=1.74)$ with $m_{Z_{d}} / \sqrt{s} \ll 1$. The significance for general values of $m_{Z_{d}} / \sqrt{s}$ and $\rho$ can be inferred from Fig. 5 with suitable rescaling of the latter parameter.

\section{ACKNOWLEDGMENTS}

We thank P. Fayet for interesting comments. This work was supported by the Ministry of Science and Technology, Taiwan under Grant No. 107-2119-M-009-017-MY3.
[1] B. Holdom, Phys. Lett. 166B, 196 (1986).

[2] P. Galison and A. Manohar, Phys. Lett. 136B, 279 (1984).

[3] R. Foot, Int. J. Mod. Phys. D 13, 2161 (2004).

[4] D. Feldman, B. Kors, and P. Nath, Phys. Rev. D 75, 023503 (2007).

[5] N. Arkani-Hamed, D. P. Finkbeiner, T. R. Slatyer, and N. Weiner, Phys. Rev. D 79, 015014 (2009).

[6] M. Pospelov and A. Ritz, Phys. Lett. B 671, 391 (2009).

[7] For a recent review, see J. Alexander et al., arXiv: 1608.08632 .
[8] K. S. Babu, C. F. Kolda, and J. March-Russell, Phys. Rev. D 57, 6788 (1998).

[9] H. Davoudiasl, H. S. Lee, and W. J. Marciano, Phys. Rev. D 85, 115019 (2012).

[10] H. Davoudiasl, H. S. Lee, I. Lewis, and W. J. Marciano, Phys. Rev. D 88, 015022 (2013).

[11] C. Boehm and P. Fayet, Nucl. Phys. B683, 219 (2004).

[12] N. Borodatchenkova, D. Choudhury, and M. Drees, Phys. Rev. Lett. 96, 141802 (2006).

[13] P. Fayet, Phys. Rev. D 75, 115017 (2007). 
[14] B. Batell, M. Pospelov, and A. Ritz, Phys. Rev. D 79, 115008 (2009).

[15] R. Essig, P. Schuster, and N. Toro, Phys. Rev. D 80, 015003 (2009).

[16] M. Reece and L. T. Wang, J. High Energy Phys. 07 (2009) 051.

[17] R. Essig, J. Mardon, M. Papucci, T. Volansky, and Y. M. Zhong, J. High Energy Phys. 11 (2013) 167.

[18] M. Karliner, M. Low, J. L. Rosner, and L. T. Wang, Phys. Rev. D 92, 035010 (2015).

[19] T. Araki, S. Hoshino, T. Ota, J. Sato, and T. Shimomura, Phys. Rev. D 95, 055006 (2017).

[20] M. He, X. G. He, and C. K. Huang, Int. J. Mod. Phys. A 32, 1750138 (2017).

[21] M. He, X. G. He, C. K. Huang, and G. Li, J. High Energy Phys. 03 (2018) 139.

[22] J. Jiang, H. Yang, and C. F. Qiao, Eur. Phys. J. C 79, 404 (2019).

[23] D. Babusci et al. (KLOE-2 Collaboration), Phys. Lett. B 736, 459 (2014).

[24] J. P. Lees et al. (BABAR Collaboration), Phys. Rev. Lett. 113, 201801 (2014).

[25] A. Anastasi et al., Phys. Lett. B 750, 633 (2015).

[26] A. Anastasi et al. (KLOE-2 Collaboration), Phys. Lett. B 757, 356 (2016).

[27] M. Ablikim et al. (BESIII Collaboration), Phys. Lett. B 774, 252 (2017).

[28] A. Anastasi et al. (KLOE-2 Collaboration), Phys. Lett. B 784, 336 (2018).

[29] J. P. Lees et al. (BABAR Collaboration), Phys. Rev. Lett. 119, 131804 (2017).

[30] J. C. Ward, Phys. Rev. 78, 182 (1950); Y. Takahashi, Nuovo Cimento 6, 371 (1957).

[31] E. Kou et al. (Belle-II Collaboration), Prog. Theor. Exp. Phys. (2019), 123C01; (2020), 029201(E).

[32] T. Abe et al. (Belle-II Collaboration), arXiv:1011.0352.

[33] J. Brodzicka et al. (Belle Collaboration), Prog. Theor. Exp. Phys. 2012, 04D001 (2012).
[34] A. J. Bevan et al. (BABAR and Belle Collaborations), Eur. Phys. J. C 74, 3026 (2014).

[35] J. L. Feng, B. Fornal, I. Galon, S. Gardner, J. Smolinsky, T. M. P. Tait, and P. Tanedo, Phys. Rev. Lett. 117, 071803 (2016).

[36] J. L. Feng, B. Fornal, I. Galon, S. Gardner, J. Smolinsky, T. M. P. Tait, and P. Tanedo, Phys. Rev. D 95, 035017 (2017).

[37] J. L. Feng, T. M. P.Tait, and C. B. Verhaaren, Phys. Rev. D 102, 036016 (2020).

[38] A. J. Krasznahorkay et al., Phys. Rev. Lett. 116, 042501 (2016).

[39] A. J. Krasznahorkay, M. Csatls, L. Csige, J. Gulys, M. Koszta, B. Szihalmi, J. Timr, D. S. Firak, A. Nagy, N. J. Sas, and G. Cern, arXiv:1910.10459.

[40] J. Jiang, L. B. Chen, Y. Liang, and C. F. Qiao, Eur. Phys. J. C 78, 456 (2018).

[41] I. Alikhanov and E. A. Paschos, Phys. Rev. D 97, 115004 (2018).

[42] P. Fayet, Phys. Lett. 69B, 489 (1977).

[43] P. Fayet, Phys. Lett. 96B, 83 (1980).

[44] M. A. Bouchiat and C. C. Bouchiat, Phys. Lett. 48B, 111 (1974).

[45] C. Bouchiat, Proc. Workshop on Neutral Current Interactions in Atoms (Cargèse, France, September 1979) (University of Michigan Press, Ann Arbor, MI, 1979).

[46] M. Abdullah, J. B. Dent, B. Dutta, G. L. Kane, S. Liao, and L. E. Strigari, Phys. Rev. D 98, 015005 (2018).

[47] M. Tanabashi et al. (Particle Data Group), Phys. Rev. D 98 , 030001 (2018).

[48] I. Adachi, T. E. Browder, P. Križan, S. Tanaka, and Y. Ushiroda (Belle-II Collaboration), Nucl. Instrum. Methods Phys. Res., Sect. A 907, 46 (2018).

[49] A. Belyaev, N. D. Christensen, and A. Pukhov, Comput. Phys. Commun. 184, 1729 (2013).

[50] For a comprehensive review, see M. Davier, A. Hocker, and Z. Zhang, Rev. Mod. Phys. 78, 1043 (2006). 